\title{
Frontières
}

\section{Porter le deuil}

\section{Diane Laflamme}

Volume 16, numéro 2, printemps 2004

Deuil, blessure vive

URI : https://id.erudit.org/iderudit/1074106ar

DOI : https://doi.org/10.7202/1074106ar

Aller au sommaire du numéro

Éditeur(s)

Université du Québec à Montréal

ISSN

1180-3479 (imprimé)

1916-0976 (numérique)

Découvrir la revue

Citer ce document

Laflamme, D. (2004). Porter le deuil. Frontières, 16(2), 3-5.

https://doi.org/10.7202/1074106ar

Ce document est protégé par la loi sur le droit d'auteur. L'utilisation des services d'Érudit (y compris la reproduction) est assujettie à sa politique d'utilisation que vous pouvez consulter en ligne.

https://apropos.erudit.org/fr/usagers/politique-dutilisation/
Cet article est diffusé et préservé par Érudit.

Érudit est un consortium interuniversitaire sans but lucratif composé de l’Université de Montréal, l'Université Laval et l'Université du Québec à Montréal. Il a pour mission la promotion et la valorisation de la recherche. https://www.erudit.org/fr/ 


\section{PORTER LE DEUIL}

\section{Diane Laflamme, Ph.D., rédactrice en chef.}

Le mot deuil est entré dans mon vocabulaire quand j'étais encore enfant et il s'est alors présenté sans drame bruyant, sous la forme d'une simple constatation formulée dans mon entourage : «il va falloir porter le deuil ». En même temps, il était question de repos, de reposer en paix et j'ai compris que ceux qui partent ont droit au repos alors que ceux qui restent ont une tâche à porter.

Porter, c'est tout un travail!

Il y a le poids de l'événement lui-même qui vous coupe le souffle quand il vous tombe dessus, puis l'arrimage au corps qui ajuste sa respiration pour continuer d'avancer. Nous portons... et nous "passons à travers ", comme le constate à son tour Catherine St-Germain Lefebvre, une jeune femme qui a écrit il y a quelques mois à peine le témoignage placé à la suite de cet éditorial. C'est le plus souvent sans grands discours que le deuil se fait une place dans l'histoire de notre famille, de notre entourage. Pas de déclarations éclatantes de portée philosophique ou sociale! Seulement les mots de tous les jours pour dire l'indicible, la perte irremplaçable, la blessure vive.

Nous ne savons pas vraiment dire la perte, nous apprenons à porter. Il arrive aussi qu'un silence qui bâillonne la parole ajoute son poids à la charge, fasse le vide autour de l'endeuillé. Ignorés ou chaleureusement entourés dans nos deuils, nous sommes des «migrants », pour reprendre le titre de l'œuvre de Derouin placée en page couverture. Porter et avancer, selon nos capacités: c'est notre lot. Heureusement, le territoire, nos territoires nous portent inlassablement, à mesure que nous avançons dans nos migrations. La vie nous traverse, sait nous trouver là où nous sommes, même ployés sous des charges inhumaines. La vie déploie ses saisons, rudes ou chatoyantes en nous et autour de nous. Il nous suffit d'avancer pas à pas, réticents ou charmés, à la fois respiration et ancrage. Transformés par la charge, mais obstinément vivants!

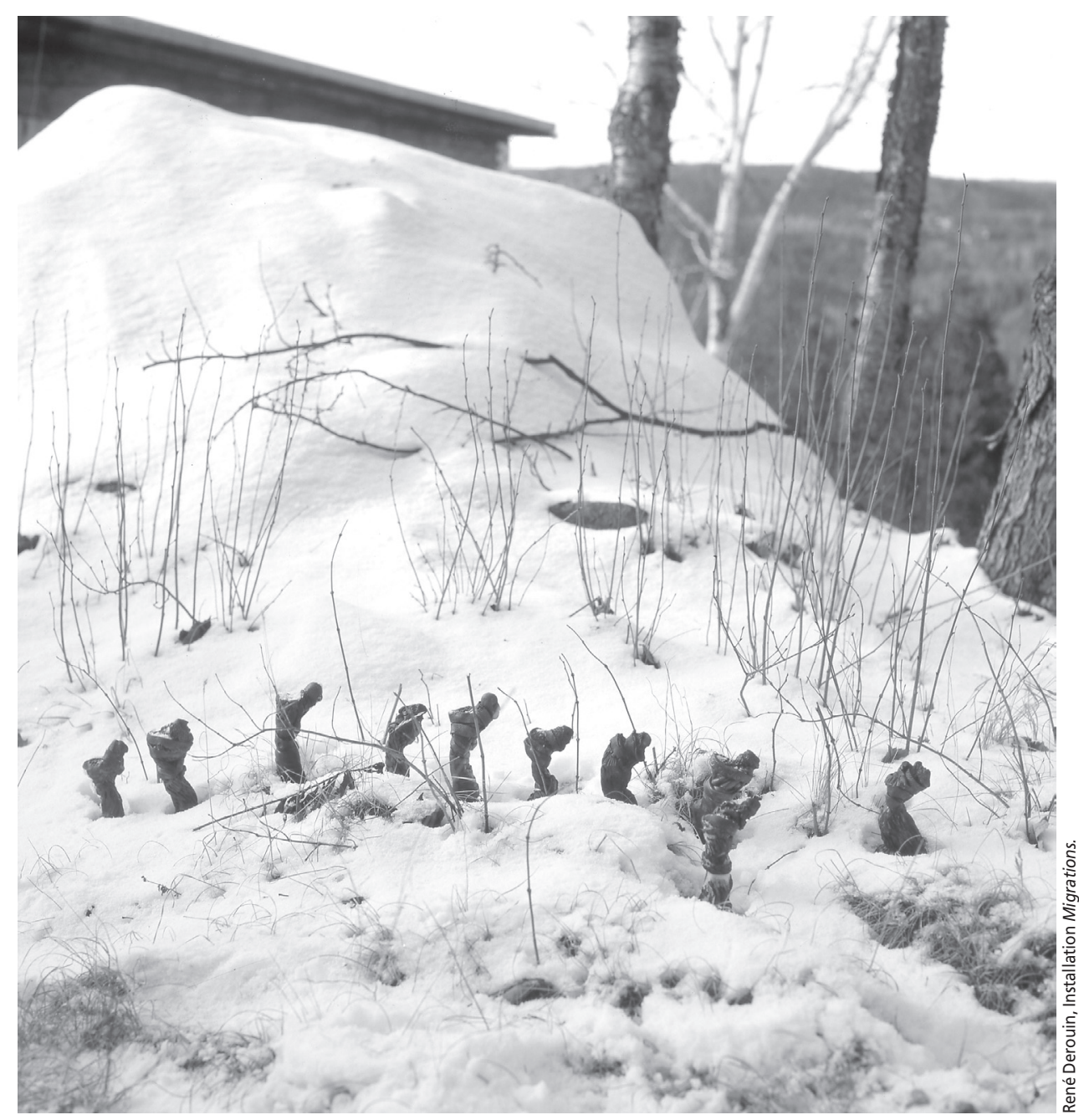




\section{DEUIL, BLESSURE VIVE}

Dans ce numéro de Frontières, le premier texte qui ouvre chacune des rubriques Article, Recherche et Intervention traite d'un même sujet, d'un deuil particulièrement lourd à porter et dont les cicatrices souvent invisibles résistent au temps et aux silences : le deuil qui fait suite à la mort d'un bébé ou d'un jeune enfant. Trois regards différents nous sont proposés, chacun contribuant à éclairer l'autre. Christiane Robitaille a travaillé de concert avec des membres de sa famille pour reconstituer une histoire au tissage bien serré où il $\mathrm{y}$ a place pour tous ses frères et sœurs, une histoire marquée par la présence indélébile d'enfants dont le passage dans ce foyer a été trop bref mais qui n'ont jamais été oubliés. Les hypothèses qu'elle propose sur le travail de deuil émergent directement de ce récit, qui fera probablement écho à ce qu'ont connu plusieurs de nos lecteurs qui ont vécu les années 1950 au Québec, en dehors des grands centres urbains.

La vie peut-elle encore avoir un sens quand on perd son enfant? Chantal Verdon vient de terminer une recherche qui a permis d'interroger à ce sujet une centaine de couples qui avaient perdu leur bébé pendant la grossesse, lors de l'accouchement ou à peine quelques jours ou quelques semaines après sa naissance. Cette étude descriptive menée dans sept hôpitaux visait à identifier les indicateurs de santé susceptibles de renseigner les intervenants sur l'intensité des réactions de deuil et à mieux comprendre l'expérience des parents afin de leur apporter le soutien voulu. Il y a au Québec des équipes de soins qui tentent de trouver des approches de plus en plus nuancées pour accompagner la personne, le couple, la famille qui viennent de perdre un enfant. Le texte de Susy Fréchette-Piperni décrit le suivi de deuil proposé depuis 1991 au Centre hospitalier Pierre-Boucher à Longueuil. Plus de 450 familles ont eu accès à cet accompagnement offert par l'équipe des infirmières du Service de natalité et on a constaté que ce besoin pouvait émerger encore des mois, voire des années après la perte.

Les liens à l'intérieur d'un couple, à l'intérieur d'une famille peuvent devenir très vulnérables lorsqu'une perte rend les partenaires inconsolables. Denise Badeau nous guide dans le dédale des difficultés liées à l'expression de la sexualité et met des mots sur les deuils trop souvent silencieux auxquels tant de femmes et d'hommes doivent faire face. Michel Pozo attire notre attention sur une autre dyade : celle qui en vient à se créer entre un grand malade et la personne qui, dans la famille, se tient au plus près, devient la plus significative pour lui. C'est un travail de deuil qui s'engage alors, même avant le décès. Le cas de l'enfant adulte qui prend soin d'un parent vieillissant affligé de démence en donne un exemple poignant.

Le deuil peut aussi prendre des dimensions transgénérationnelles. Hélène de la Vaissière en trouve un exemple éloquent dans sa pratique auprès de jeunes délinquants issus de familles émigrées en France. C'est là un aspect du deuil fréquemment oublié car les questions identitaires sont le plus souvent abordées en faisant référence au vécu individuel de la personne plutôt qu'à son insertion dans un territoire. On peut découvrir des résonances entre ces propos et la quête de soi dont traite René Derouin quand il décrit la grande migration qui a fait de lui un artiste ancré dans le territoire des Amériques.

Le deuil constitue un drame privé à maints égards, mais le réseau social auquel participent les endeuillés ne reste pas neutre lors de l'événement de la perte et contribue à alourdir la peine ou à l'alléger. Certains endeuillés voient le vide se faire autour d'eux; d'autres sont assiégés par une meute de présences non sollicitées qui s'imposent dans leur univers. Nos auteurs se penchent sur les circonstances qui peuvent provoquer ces deux catégories de deuils complexes, que Lucie Fréchette appelle les deuils "surexposés » et les deuils "cachés ou clandestins». Le drame des endeuillés peut être propulsé malgré eux sur la place publique à la suite d'une intervention des officiers de l'État ou des médias. À l'autre extrémité du spectre, le groupe social restera sourd à la souffrance que provoquent les deuils dont il ne veut pas entendre parler, refusera tout appui aux endeuillés dont il ne veut pas voir les blessures. Redoublant d'attention pour mieux identifier les modes d'intervention les plus appropriés, Lucie Fréchette se joint à Amnon Jacob Suissa pour signer un autre texte analysant plus en profondeur le deuil des familles affligées par un décès survenu en contexte de dépendance.

Mal aimés ou trop aimés lorsqu'ils quittent leur place parmi nous, les grands de ce monde ont retenu l'attention de Joseph J. Lévy et Isabelle Toupin. Leur article nous permet d'observer ce qui se joue sur la scène médiatique et s'attarde plus particulièrement aux messages placés sur des sites Internet lors du décès de Lady Di. Au-delà des frontières qu'imposent la géographie et les classes sociales, toutes ces personnes déclarent porter un même deuil et tiennent à en témoigner par écrit. Invoquer le secours des mots quand le deuil est lourd à porter, c'est aussi ce qu'ont fait plusieurs écrivains. Hélène Reboul nous présente quelques-unes de ces œuvres littéraires et nous invite à parcourir avec elle ces écrits de deuil. Dans un autre registre et en fouillant dans des œuvres fort anciennes, Jean-Jacques Lavoie a rassemblé des paroles de deuil qui donnent une place inattendue à nos compagnons les animaux et qui disent jusqu'à quel point l'espèce humaine n'a pas le monopole de la souffrance lorsque la mort est passée. Cette recherche menée à partir des textes nous ramène d'abord à l'Antiquité pour mieux nous replonger dans notre présent et nous rappeler à une solidarité avec tous les êtres sensibles.

À la rubrique Regard, d'autres blessures, d'autres deuils encore cherchent pour se dire non plus seulement des mots mais des cris, des conjurations et des chansons. Anne Santorin nous parle d'une histoire d'amour et de mort. Elle retrace pour nous, dans la poésie des chansons de Jacques Michel, le patient cheminement d'un travail de deuil en quatre saisons qui s'exprime dans des musiques qui nous sont familières. Martyne-Isabel Forest bouleverse les conventions intergénérationnelles pour faire sa juste place à une peine qui explore ses propres chemins, qui respecte ses propres attachements. Ce parcours lucide parle d'amour. Il y a une femme qui s'est faite grand-mère et enchanteresse pour qu'une enfant puisse se blottir dans ses mots et dans ses gestes.

Les mots et les gestes peuvent aussi se faire rites de guérison et apaiser la blessure du deuil. De nos jours, les voies d'une éventuelle guérison par la ritualité sont peu balisées. Les rites de deuil n'obéissent plus nécessairement à une codification dictée par des instances sociales ou des traditions religieuses habituées à tout prévoir pour assurer un prompt retour à l'ordre normal des choses. La démarche que décrivent Nicole Bouchard et Mario Bélanger nous fait découvrir un partenariat novateur avec une entreprise funéraire de la région du Saguenay-Lac-Saint-Jean et confirme jusqu'à quel point l'émergence d'une ritualité renouvelée vient combler un besoin indéniable.

Qu'il s'agisse d'explorer les contextes dans lesquels se vit le deuil, ou encore de trouver les mots et les gestes pour 
dire ce deuil, constamment les textes proposés dans ce numéro s'interrogent sur la qualité de la présence à autrui. Deux articles abordent de l'intérieur l'univers des intervenants, ces personnes qui portent leurs propres deuils et qui aident d'autres à porter leur fardeau. Johanne de Montigny a été en mesure d'observer dans les milieux de soins palliatifs comment la perte d'un proche peut jouer le rôle d'élément déclencheur pour amener l'endeuillé à se proposer ensuite pour accompagner autrui en qualité d'intervenant professionnel, de personne significative auprès d'un grand malade ou de bénévole. C'est là une expérience «troublante», dit-elle, lorsque «l'épreuve de la perte dicte l'envie de soigner », lorsque «la perte agit comme le détonateur de la compassion ». Pierre Migneault nous offre pour sa part un témoignage sans détour qui s'alimente à une longue pratique du deuil de soi et de l'accompagnement de ce deuil de soi dans l'intervention psychothérapeutique. De pièges en tentations, le parcours qu'il nous invite à réaliser avec lui « en amont comme en aval de l'intervention » nous fait découvrir des paysages concrets et des ambiances mythiques inattendus. De ses vignettes cliniques et de son placard à suicides émergent des réflexions, des gestes habités par un même entêtement que l'on souhaite contagieux: «y retrouver son âme et son bon sens ».

\section{LARGAGE ET RESSAC}

Signalons enfin qu'avec ce numéro Frontières vient s'inscrire à son tour dans le vaste mouvement de ressac ${ }^{1}$ déclenché par l'artiste René Derouin lorsqu'il a largué aux quatre vents et dans deux cent cinquante mains les statuettes de son œuvre Migrations. Que portent-elles ces figurines qui ont marché l'Amérique tant au nord qu'au sud, et qui traversent maintenant nos pages? Comment leur pesante migration se joindra-t-elle à la nôtre aujourd'hui, à mesure qu'elles traceront leur chemin d'une page à l'autre, mêlées à la respiration des textes de nos auteurs? Ramassés sous le poids de leur argile, ces personnages poursuivent leur route, portés ici encore par un nouveau territoire.

Porter ses morts en terre, porter le deuil, c'est aussi un apprentissage de la dignité. Un fardeau dont on accepte le poids comme on accepte de prendre sa place dans une histoire. Même blessé à vif, même écrasé sous la charge, avancer... En se disant que notre respiration haletante en viendra bien à passer à travers, car nous ne sommes que souffle.

\section{Note}

1. Sous le titre Ressac, René Derouin a publié à l'Hexagone en 1996 un livre dans lequel il décrit le cheminement qui l'a conduit à larguer dans le fleuve Saint-Laurent 19000 des figurines de son œuvre Migrations; il a aussi rassemblé dans un chapitre les textes qu'il a reçus après le largage de 250 figurines «en milieu culturel » (Chap.V, p. 86-167). Le largage de cette œuvre continue encore de provoquer des ressacs, d'inspirer d'autres créateurs. Mentionnons par exemple France Ducasse, qui vient de publier aux éditions l'Instant même un recueil de nouvelles intitulé La Mort ne tue personne, dont le dernier texte, "Le don de la mort », est dédié à René Derouin et fait référence au largage des 19000 figurines. 